\title{
ON THE ASYMPTOTIC BEHAVIOR OF THE ONE-DIMENSIONAL MOTION OF THE POLYTROPIC IDEAL GAS WITH STRESS-FREE CONDITION*
}

BY

\section{TAKEYUKI NAGASAWA}

Keio University, Yokohama, Japan ${ }^{1}$

1. Introduction. We consider the following initial-boundary value problem:

$$
\begin{gathered}
u_{t}=v_{x}, \\
v_{t}=\left(-R \frac{\theta}{u}+\mu \frac{v_{x}}{u}\right)_{x}, \\
c_{V} \theta_{t}=-R \frac{\theta v_{x}}{u}+\mu \frac{v_{x}^{2}}{u}+\kappa\left(\frac{\theta_{x}}{u}\right)_{x},
\end{gathered}
$$

for $(x, t) \in[0,1] \times[0,+\infty)$ with the initial condition

$$
(u, v, \theta)(x, 0)=\left(u_{0}, v_{0}, \theta_{0}\right)(x), \quad u_{0}>0, \quad \theta_{0}>0,
$$

and the boundary condition

$$
\begin{aligned}
\left(-R \frac{\theta}{u}+\mu \frac{v_{x}}{u}\right)(0, t) & =\left(-R \frac{\theta}{u}+\mu \frac{v_{x}}{u}\right)(1, t)=0, \\
\theta_{x}(0, t) & =\theta_{x}(1, t)=0 .
\end{aligned}
$$

This problem is a model of the one-dimensional motion of the polytropic ideal gas with adiabatic ends which is put into a vacuum. $(u, v, \theta)$, unknown functions, represent the specific volume, the velocity, the absolute temperature of the gas; $\left(R, \mu, c_{V}, \kappa\right)$, given positive constants, stand for the gas constant, the coefficient of viscosity, the heat capacity at constant volume, and the coefficient of heat conduction, respectively. The condition (1.5) is called the stress-free condition.

Kazhykhov showed the global existence of a unique solution to this problem in [2]. He constructed the solution $(u, v, \theta)$ in the Hölder class $\bigcap_{T>0}\left\{B_{T}^{1+\alpha} \times H_{T}^{2+\alpha} \times H_{T}^{2+\alpha}\right\}$ $(0<\alpha<1)$ provided $\left(u_{0}, v_{0}, \theta_{0}\right)$ belongs to $H^{1+\alpha} \times H^{2+\alpha} \times H^{2+\alpha}$. (For the definition of the Hölder spaces $H^{n+\alpha}$ etc., see [3].) We call this solution classical in this paper.

More recently Okada [5] and Kawashima [1] showed the asymptotic behavior of the solution. The problem has a trivial solution

$$
u(x, t)=\bar{u}(1+t), \quad v(x, t)=\bar{u}\left(x-\frac{1}{2}\right), \quad \theta(x, t)=\bar{\theta},
$$

${ }^{*}$ Received May 15, 1987.

'Present address: Tôhoku University, Sendai, Japan. 
corresponding to initial data

$$
u_{0}(x)=\bar{u}, \quad v_{0}(x)=\bar{u}\left(x-\frac{1}{2}\right), \quad \theta_{0}(x)=\bar{\theta},
$$

where $\bar{u}$ and $\bar{\theta}$ are positive constants satisfying the relation

$$
\mu \bar{u}=R \bar{\theta} .
$$

In [5] and [1], they proved any classical solution which satisfies some restricted assumptions on the initial data and/or the ratio between $R$ and $c_{V}$ converges to the state like (1.7).

On the other hand, the author has already investigated in [3] other asymptotic properties of the solution which give the growth of $u$ and $\int_{0}^{1} u d x$ without the restricted assumptions.

In this paper the author attempts to show the convergence of the classical solution and its rate without any restricted assumptions. We have the following result.

THEOREM 1.1. Let $(\bar{u}, \bar{\theta})$ be a positive root of the simultaneous equations (1.8) and

$$
\int_{0}^{1}\left(\frac{1}{2} v_{0}^{2}(x)+c_{V} \theta_{0}(x)\right) d x=\int_{0}^{1} \frac{1}{2}\left\{\int_{0}^{1} v_{0}(x) d x+\bar{u}\left(x-\frac{1}{2}\right)\right\}^{2} d x+c_{V} \bar{\theta} .
$$

Then there exist positive constants $\lambda$ and $C$ which depend on $R, \mu, c_{V}, \kappa$, and the initial data but not on $t$ such that the classical solution $(u, v, \theta)$ to the problem (1.1)(1.6) satisfies the estimate

$$
\left\|\left(\frac{u(x, t)}{1+t}-\bar{u}, v(x, t)-\int_{0}^{1} v_{0}(x) d x-\bar{u}\left(x-\frac{1}{2}\right), \theta(x, t)-\bar{\theta}\right)\right\|_{1.2}^{2} \leq C(1+t)^{-\lambda} .
$$

Here $\|\cdot\|_{1,2}$ is the norm of the Sobolev space $W^{1,2}(0,1)$.

Remark. A positive root $(\bar{u}, \bar{\theta})$ of the above simultaneous equations exists; $\bar{u}$ and $\bar{\theta}$ are given by

$$
\left\{\begin{array}{l}
\bar{u}=\frac{2}{R}\left[\sqrt{36 c_{V}^{2} \mu^{2}+3 R^{2}\left\{\left(2 E_{0}-\left(\int_{0}^{1} v_{0}(x) d x\right)^{2}\right\}\right.}-6 c_{V} \mu\right], \\
\bar{\theta}=\frac{2 \mu}{R^{2}}\left[\sqrt{36 c_{V}^{2} \mu^{2}+3 R^{2}\left\{2 E_{0}-\left(\int_{0}^{1} v_{0}(x) d x\right)^{2}\right\}}-6 c_{V} \mu\right], \\
E_{0}=\int_{0}^{1}\left(\frac{1}{2} v_{0}^{2}(x)+c_{V} \theta_{0}(x)\right) d x .
\end{array}\right.
$$

We shall show the convergence $\left(u /(1+t), v-\int_{0}^{1} v_{0}(x) d x-\bar{u}\left(x-\frac{1}{2}\right), \theta\right)$ to $(\bar{u}, 0, \bar{\theta})$ in Section 2, and its rate in Section 3. The idea of the proof is that we transform the original problem $(1.1)-(1.6)$ to the reduced problem (2.5)-(2.7) with (2.9)-(2.11) below by the changes of unknown functions and the time variable. We shall study the asymptotics of the latter problem. 
2. Convergence of solution. In order to prove Theorem 1.1 , it is convenient to transform the problem into the one somewhat similar to the outer pressure problem which was discussed in [4]. First we change an unknown function $u \rightarrow \tilde{u}=u /(1+t)$, and then change a variable $t \rightarrow \hat{t}=\log (1+t)$. Thus we can rewrite (1.1)-(1.3) as

$$
\begin{gathered}
\hat{\tilde{u}}_{\hat{t}}+\hat{\tilde{u}}=\hat{v}_{x}, \\
\hat{v}_{\hat{t}}=\left(-R \frac{\hat{\theta}}{\hat{\tilde{u}}}+\mu \frac{\hat{v}_{x}}{\hat{\tilde{u}}}\right)_{x}, \\
c_{V} \hat{\theta}_{\hat{t}}=-R \frac{\hat{\theta} \hat{v}_{x}}{\hat{\tilde{u}}}+\mu \frac{\hat{v}_{x}^{2}}{\hat{\tilde{u}}}+\kappa\left(\frac{\hat{\theta}_{x}}{\hat{\tilde{u}}}\right)_{x} .
\end{gathered}
$$

Here we use the notation $\hat{f}$ to mean

$$
\hat{f}=\hat{f}(x, \hat{t})=f(x, t(\hat{t}))=f\left(x, e^{\hat{i}}-1\right)
$$

for a function $f(x, t)$ of $x$ and $t$. However, to avoid complicated notation, in what follows, we write again $(\hat{\tilde{u}}, \hat{v}, \hat{\theta}, \hat{t})$ as $(u, v, \theta, t)$. Moreover, we introduce a new unknown function

$$
w(x, t)=v(x, t)-\int_{0}^{1} v_{0}(\xi) d \xi-\int_{0}^{x} u(\xi, t) d \xi+\int_{0}^{1} \int_{0}^{\xi} u(\eta, t) d \eta d \xi .
$$

If we use $w(x, t)$ instead of $v(x, t)$, the boundary condition (1.5) will be transformed into (2.10), which is the same type as that of the outer pressure problem. As the outer pressure problem was discussed in [4], we improve its arguments to derive our result.

Remark that $w$ belongs to $\bigcap_{T>0} H_{T}^{2+\alpha}$ if $(u, v)$ belongs to $\bigcap_{T>0}\left\{B_{T}^{1+\alpha} \times H_{T}^{2+\alpha}\right\}$. Using $w(x, t)$, we can deduce $(2.1)-(2.3)$ as follows:

$$
\begin{gathered}
u_{t}=w_{x}, \\
w_{t}+w=\left(-R \frac{\theta}{u}+\mu \frac{w_{x}}{u}\right)_{x}, \\
c_{V} \theta_{t}=\mu w_{x}+\mu u-R \theta+\frac{\left(\mu w_{x}+\mu u-R \theta\right) w_{x}}{u}+\kappa\left(\frac{\theta_{x}}{u}\right)_{x} .
\end{gathered}
$$

In rewriting (2.6), we use the identity

$$
\int_{0}^{1} v(x, t) d x=\int_{0}^{1} v_{0}(x) d x
$$

which follows easily from (1.2) and (1.5).

Initial and boundary conditions (1.4)-(1.6) are deduced:

$$
\begin{gathered}
(u, w, \theta)(x, 0)=\left(u_{0}, w_{0}, \theta_{0}\right)(x), \quad u_{0}>0, \quad \theta_{0}>0, \quad \int_{0}^{1} w_{0} d x=0, \\
\left(-R \frac{\theta}{u}+\mu \frac{w_{x}}{u}\right)(0, t)=\left(-R \frac{\theta}{u}+\mu \frac{w_{x}}{u}\right)(1, t)=-\mu, \\
\theta_{x}(0, t)=\theta_{x}(1, t)=0 .
\end{gathered}
$$


Since the original problem (1.1)-(1.6) has the solution in $\bigcap_{T>0}\left\{B_{T}^{1+\alpha} \times H_{T}^{2+\alpha} \times\right.$ $\left.H_{T}^{2+\alpha}\right\}$, the reduced problem (2.5)-(2.7) with (2.9)-(2.11) also has a global solution in the same class. Moreover both $u$ and $\theta$ are positive $([2,3])$. In the sequel we shall investigate the asymptotic properties of the solution $(u, w, \theta)$ to the reduced problem. In this section we shali prove

TheOREM 2.1. The classical solution $(u, w, \theta)$ to the initial-boundary value problem (2.5)-(2.7), (2.9)-(2.11) converges to $(\bar{u}, 0, \bar{\theta})$ in $W^{1,2}(0,1)$ as $t \rightarrow+\infty$.

This theorem says, by use of the terminology of the original problem (1.1)-(1.6), that $\left(u /(1+t), v-\int_{0}^{1} v_{0} d x-\bar{u}\left(x-\frac{1}{2}\right), \theta\right)$ converges to $(\bar{u}, 0, \bar{\theta})$ in $W^{1,2}(0,1)$.

The proof of Theorem 2.1 is divided into three steps. Firstly we show the uniform (with respect to $x)$ convergence of $u$ to $\bar{u}$, secondly the convergence $(w, \theta)$ to $(0, \bar{\theta})$ in $L^{2}(0,1)$, lastly the decay of derivatives of the solution in $L^{2}(0,1)$.

1 st Step. Uniform convergence of $u$ to $\bar{u}$. Since $\bar{u}$ is a positive root of the quadratic equation

$$
\bar{u}^{2}+\frac{24 c_{V} \mu}{R} \bar{u}+12\left(\int_{0}^{1} v_{0}(x) d x\right)^{2}-24 E_{0}=0,
$$

in order to show the convergence of $u$, it is enough to see

Proposition 2.1. $u(x, t)$ satisfies

$$
u^{2}(x, t)+\frac{24 c_{V} \mu}{R} u(x, t)+12\left(\int_{0}^{1} v_{0}(x) d x\right)^{2}-24 E_{0}=o(1),
$$

where $o(1)$ denotes the function which converges to zero uniformly in $x \in[0,1]$ as $t \rightarrow+\infty$.

Because the proof of this proposition is lengthy, first we mention its outline and then give it by some lemmas.

Outline of Proof. We integrate (2.6) over $[0, x]$ by use of $(2.10)$ to get

$$
\frac{\partial}{\partial t} \int_{0}^{x} w(\xi, t) d \xi+\int_{0}^{x} w(\xi, t) d \xi=\frac{-R \theta+\mu w_{x}+\mu u}{u} .
$$

Multiplying both sides of the result by $\mu^{-1} u(x, t)$ and using (2.5), we have

$$
u_{t}(x, t)+u(x, t)\left(1-\frac{1}{\mu} \frac{\partial}{\partial t} \int_{0}^{x} w(\xi, t) d \xi\right)=\frac{R}{\mu} \theta(x, t)+\frac{1}{\mu} u(x, t) \int_{0}^{x} w(\xi, t) d \xi .
$$

Therefore we have

$$
\begin{aligned}
& u(x, t)=e^{-t} \exp \left\{\frac{1}{\mu} \int_{0}^{x}\left(w(\xi, t)-w_{0}(\xi)\right) d \xi\right\} u_{0}(x) \\
& +e^{-t} \int_{0}^{t} e^{\tau} \exp \left\{\frac{1}{\mu} \int_{0}^{x}(w(\xi, t)-w(\xi, \tau)) d \xi\right\}\left(\frac{R}{\mu} \theta(x, \tau)+\frac{1}{\mu} u(x, \tau) \int_{0}^{x} w(\xi, \tau) d \xi\right) d \tau .
\end{aligned}
$$

On the other hand, it is easy to see that our problem has the energy identity

$$
\int_{0}^{1}\left(\frac{1}{2} v^{2}(x, t)+c_{V} \theta(x, t)\right) d x \equiv E_{0}
$$


(see [3]).

Thus, we get the proposition if we show the following facts:

$$
\begin{gathered}
C^{-1} \leq u(x, t) \leq C, \\
\int_{0}^{x} w(\xi, t) d \xi=o(1), \\
e^{-t} \int_{0}^{t} e^{\tau}\left|\theta(x, \tau)-\int_{0}^{1} \theta(x, t) d x\right| d \tau=o(1), \\
e^{-t} \int_{0}^{t} e^{\tau} \int_{0}^{1} w^{2}(x, \tau) d x d \tau=o(1), \\
\int_{0}^{1} u(x, t) d x-u(x, t)=o(1), \\
e^{-t} \int_{0}^{t} e^{\tau}\left(\int_{0}^{1} u(x, \tau) d x\right)^{2} d \tau-\left(\int_{0}^{1} u(x, t) d x\right)^{2}=o(1) .
\end{gathered}
$$

Hereafter $C(>1)$ denotes a positive constant depending on $R, \mu, c_{V}, \kappa$, and the initial data but not on $t$. Indeed, we have

$$
\begin{aligned}
u(x, t) & =e^{-t} \int_{0}^{t} e^{\tau} \int_{0}^{1} \frac{R}{\mu} \theta(x, \tau) d x d \tau+o(1) \quad \text { (by (2.13), (2.16), (2.15), (2.17)) } \\
& =e^{-t} \int_{0}^{t} e^{\tau} \frac{R}{c_{V} \mu}\left(E_{0}-\int_{0}^{1} \frac{1}{2} v^{2}(x, \tau) d x\right) d \tau+o(1) \quad \text { (by (2.14)) } \\
& =\frac{R}{c_{V} \mu}\left\{E_{0}-\frac{1}{2}\left(\int_{0}^{1} v_{0}(x) d x\right)^{2}-\frac{e^{-t}}{24} \int_{0}^{t} e^{\tau}\left(\int_{0}^{1} u(x, \tau) d x\right)^{2} d \tau\right\}+o(1) \\
& =\frac{R}{c_{V} \mu}\left\{E_{0}-\frac{1}{2}\left(\int_{0}^{1} v_{0}(x) d x\right)^{2}-\frac{1}{24} u^{2}(x, t)\right\}+o(1)
\end{aligned}
$$

LEMMA 2.1. We have (2.15).

Proof. We use the expression due to Kazhykhov [2],

$$
u(x, t)=\frac{e^{-t}}{B(x, t)}\left\{u_{0}(x)+\int_{0}^{t} \frac{R}{\mu} e^{\tau} \theta(x, \tau) B(x, \tau) d \tau\right\},
$$

where

It is crucial to show

$$
B(x, t)=\exp \left\{\frac{1}{\mu} \int_{0}^{x}\left(v_{0}(\xi)-v(\xi, t)\right) d \xi\right\} .
$$

$$
C^{-1} \leq \int_{0}^{1} \theta(x, t) d x \leq C,
$$




$$
\int_{0}^{t} \int_{0}^{1} \frac{\theta_{x}^{2}}{u \theta^{2}} d x d \tau \leq C
$$

(for detail see [4, Lemmas 4.1-4.2]).

Dividing both sides of (2.7) by $\theta$, and integrating with respect to $x$ over $[0,1]$, we have

$$
\frac{d}{d t} \int_{0}^{1} c_{V} \log \theta d x=\mathscr{V}(t)+\frac{d}{d t} \frac{R}{\mu} \int_{0}^{1} \int_{0}^{x} v(\xi, t) d \xi d x .
$$

Here we also use (2.6), (2.10), and (2.4). Integrating over $[0, t]$ and using (2.14), we have

$$
\mathscr{U}(t)+\int_{0}^{t} \mathscr{V}(\tau) d \tau \leq C,
$$

where $\mathscr{U}(t)$ and $\mathscr{V}(t)$ are nonnegative functions of $t$ defined by

$$
\begin{aligned}
& \mathscr{U}(t)=\int_{0}^{1}\left\{\frac{1}{2} v^{2}(x, t)+c_{V}(\theta(x, t)-\log \theta(x, t)-1)\right\} d x, \\
& \mathscr{V}(t)=\int_{0}^{1}\left\{\frac{\left(\mu w_{x}+\mu u-R \theta\right)^{2}}{\mu u \theta}(x, t)+\frac{\kappa \theta_{x}^{2}}{u \theta^{2}}(x, t)\right\} d x .
\end{aligned}
$$

Thus we get (2.22). In a similar way to the proof of [4, Lemma 4.1], (2.21) follows from (2.14) and (2.23).

By similar arguments to [4, Lemmas 4.1-4.2], we have the following estimate, which we need to prove (2.16).

Corollary. We have

$$
\theta(x, t) \leq C(1+\mathscr{V}(t))
$$

LeMmA 2.2. We have (2.16).

Proof. From (2.15), (2.4), and (2.14), we have

$$
\int_{0}^{1} w^{2}(x, t) d x \leq C
$$

Therefore it is sufficient to see

$$
\int_{0}^{1}\left(\int_{0}^{x} w(\xi, t) d \xi\right)^{2} d x=o(1) .
$$

After multiplying both sides of $(2.12)$ by $\int_{0}^{x} w(\xi, t) d \xi$, we integrate with respect to $x$ over $[0,1]$. Taking account of $(2.14),(2.25)$, and $(2.26)$, we have

$$
\frac{d}{d t} \int_{0}^{1}\left(\int_{0}^{x} w(\xi, t) d \xi\right)^{2} d x+\int_{0}^{1}\left(\int_{0}^{x} w(\xi, t) d \xi\right)^{2} d x \leq C \mathscr{V}(t) .
$$

Consequently the integrability $(2.24)$ of $\mathscr{V}(t)$ yields the desired assertion.

(2.24) gives (2.17) also. 
Lemma 2.3. We have (2.17)-(2.19).

Proof. It follows from (2.15), (2.25), (2.21), and the definition of $\mathscr{V}(t)$ that

$$
\begin{aligned}
& \left|\int_{0}^{1} \theta(x, t) d x-\theta(x, t)\right| \\
& \quad \leq\left\{\int_{0}^{1} \frac{\theta_{x}^{2}}{u \theta^{2}} d x \cdot \int_{0}^{1} \theta(x, t) d x \cdot \sup _{(x, t) \in[0,1] \times[0,+\infty)} u(x, t) \theta(x, t)\right\}^{1 / 2} \\
& \quad \leq \varepsilon+C(\varepsilon) \mathscr{V}(t)
\end{aligned}
$$

for any $\varepsilon>0$. Thus we have (2.17).

Next we shall show (2.18). Making use of (2.13), (2.15), and (2.16), we have

$$
\int_{0}^{1} \mu u(x, t) d x-e^{-t} \int_{0}^{t} e^{\tau} \int_{0}^{1} R \theta(x, \tau) d x d \tau=o(1) .
$$

Therefore (2.18) follows if we show

$$
\int_{0}^{1} \mu u(x, t) d x-e^{-t} \int_{0}^{t} e^{\tau} \int_{0}^{1} R \theta(x, \tau) d x d \tau=e^{-t} \int_{0}^{t} e^{\tau} \int_{0}^{1} w^{2}(x, \tau) d x d \tau+o(1) .
$$

To prove this we multiply both sides of $(2.12)$ by $u(x, t)$, and integrating over $[0,1]$, we have

$$
\begin{array}{r}
\frac{d}{d t} \int_{0}^{1} u(x, t) \int_{0}^{x} w(\xi, t) d \xi d x+\int_{0}^{1} u(x, t) \int_{0}^{x} w(\xi, t) d \xi d x+\int_{0}^{1}\left(w^{2}+R \theta\right) d x \\
=\frac{d}{d t} \int_{0}^{1} \mu u d x+\int_{0}^{1} \mu u d x .
\end{array}
$$

Here we perform integration by parts with the help of $(2.5)$ and

$$
\int_{0}^{1} w(x, t) d x \equiv 0
$$

which follows from (2.8) and (2.4). By use of (2.15) and (2.16), integration of (2.28) yields the desired fact.

(2.19) is valid by (2.13), (2.15)-(2.17), and (2.27).

To prove $(2.20)$ we need

LeMma 2.4. We have

$$
\int_{0}^{1}\left(\frac{1}{2} w^{2}(x, t)+c_{V} \theta(x, t)-\frac{c_{V} \mu}{R} u(x, t)\right) d x=o(1) .
$$

Proof. Multiplying both sides of (2.6) by $w(x, t)$, adding to (2.7), and integrating with respect to $x$ over $[0,1]$, by use of boundary conditions $(2.10),(2.11)$, and Equation (2.5) we have

$$
\frac{d}{d t} \int_{0}^{1}\left(\frac{1}{2} w^{2}+c_{V} \theta\right) d x+\int_{0}^{1}\left(w^{2}+R \theta\right) d x=\frac{d}{d t} \int_{0}^{1} \mu u d x+\int_{0}^{1} \mu u d x .
$$

From (2.28) and (2.31), in a manner similar to the proof of (2.18) we get

$$
\int_{0}^{1}\left(\frac{1}{2} w^{2}(x, t)+c_{V} \theta(x, t)\right) d x-e^{-t} \int_{0}^{t} e^{\tau} \int_{0}^{1} c_{V} \theta(x, \tau) d x d \tau=o(1) .
$$

Here we use (2.18). From this and (2.27), we get the assertion. 
LEMMA 2.5. We have (2.20).

Proof. By use of (2.5), we get

$$
\begin{aligned}
& e^{-t} \int_{0}^{t} e^{\tau}\left(\int_{0}^{1} u(x, \tau) d x\right)^{2} d \tau-\left(\int_{0}^{1} u(x, t) d x\right)^{2} \\
& =e^{-t}\left(\int_{0}^{1} u_{0}(x) d x\right)^{2}-2 e^{-t} \int_{0}^{t} e^{\tau} \int_{0}^{1} u(x, \tau) d x \int_{0}^{1} w_{x}(x, \tau) d x d \tau .
\end{aligned}
$$

Clearly, the first term of the right-hand side tends to zero as $t \rightarrow+\infty$. Since $u$ is bounded, the second term is majorized as

$$
\begin{aligned}
& \left|e^{-t} \int_{0}^{t} e^{\tau} \int_{0}^{1} u(x, \tau) d x \int_{0}^{1} w_{x}(x, \tau) d x d \tau\right| \\
& \leq C e^{-t} \int_{0}^{t} e^{\tau}\left\{\int_{0}^{1}\left(\left|\mu w_{x}+\mu u-R \theta\right|+\left|u-\int_{0}^{1} u d x\right|+\left|\theta-\int_{0}^{1} \theta d x\right|+w^{2}\right) d x\right. \\
& \left.+\left|\int_{0}^{1}\left(\frac{1}{2} w^{2}+c_{V} \theta-\frac{c_{V} \mu}{R} u\right) d x\right|\right\} d \tau .
\end{aligned}
$$

The right-hand side tends to zero as $t \rightarrow+\infty$ because of (2.24), (2.15), (2.21), (2.19), (2.17), (2.18), and (2.30).

2 nd Step. $L^{2}(0,1)$ convergence of $(w, \theta)$ to $(0, \bar{\theta})$. Since we have shown the uniform convergence of $u$, the $L^{2}$-decay of $w$ implies the convergence of $\int_{0}^{1} \theta d x$ to $\bar{\theta}$ by virtue of (2.30). Thus our aim in this step is accomplished if we show

Proposition 2.2. For any $k>0$,

$$
\begin{aligned}
\int_{0}^{1}\left[\left\{\frac{1}{2} w^{2}(x, t)+c_{V}\right.\right. & \left.\left.\left(\theta(x, t)-\int_{0}^{1} \theta(x, t) d x\right)\right\}^{2}+w^{4}(x, t)\right] d x \\
+e^{-k t} \int_{0}^{t} e^{k \tau} & \left\{\int_{0}^{1}\left(\theta_{x}^{2}(x, \tau)+w^{2}(x, \tau) w_{x}^{2}(x, \tau)\right) d x\right. \\
+ & \left.\int_{0}^{1} w^{2}(x, \tau) d x \cdot \int_{0}^{1} w_{x}^{2}(x, \tau) d x\right\} d \tau=o(1)
\end{aligned}
$$

holds.

Before proving this proposition, we must show the following lemma, which is an extension of (2.18).

LEMMA 2.6. We have

$$
e^{-k t} \int_{0}^{t} e^{k \tau} \int_{0}^{1} w^{2}(x, \tau) d x d \tau=o(1)
$$

for any $k>0$.

Proof. It is clear for $k \geq 1$ because of (2.18). To prove for $0<k<1$, we multiply both sides of $(2.12)$ by $u(x, t) \int_{0}^{x} w(\xi, t) d \xi$, and perform integration by parts with 
respect to $x$ over $[0,1]$ with the help of $(2.5)$ and (2.29). Then, by use of the estimates in the previous step, one gets

$$
\begin{array}{r}
\frac{d}{d t} \int_{0}^{1} u(x, t)\left(\int_{0}^{x} w(\xi, t) d \xi\right)^{2}+2 \int_{0}^{1} u(x, t)\left(\int_{0}^{x} w(\xi, t) d \xi\right)^{2}+2 \mu \int_{0}^{1} w^{2}(x, t) d x \\
\leq C \max _{x \in[0,1]}\left|\int_{0}^{x} w(\xi, t) d \xi\right|
\end{array}
$$

Therefore the integration of the above differential inequality yields the assertion for $0<k<2$. Here we use (2.16).

Proof of Proposition 2.2. By use of (2.6), (2.7), (2.10), and (2.11), we have

$$
\begin{aligned}
\frac{\partial}{\partial t}\left\{\frac{1}{2} w^{2}+c_{V}\left(\theta-\int_{0}^{1} \theta d x\right)\right\}+w^{2} & \\
=\mu w_{x}+\mu u- & R \theta+\frac{\partial}{\partial x}\left[-R \frac{\theta w}{u}+\mu \frac{w w_{x}}{u}+\mu w+\kappa \frac{\theta_{x}}{u}\right] \\
& \quad \int_{0}^{1}\left(\mu w_{x}+\mu u-R \theta-R \frac{\theta w_{x}}{u}+\mu \frac{w_{x}^{2}}{u}+\mu w_{x}\right) d x .
\end{aligned}
$$

Remark that terms in brackets vanish at $x=0$ and 1 by (2.10) and (2.11). We multiply both sides by $\frac{1}{2} w^{2}+c_{V}\left(\theta-\int_{0}^{1} \theta d x\right)$ and integrate with respect to $x$ over $[0,1]$. Thus we get

$$
\begin{aligned}
\frac{1}{2} \frac{d}{d t} \int_{0}^{1}\left\{\frac{1}{2} w^{2}+c_{V}\left(\theta-\int_{0}^{1} \theta d x\right)\right\}^{2} d x+\int_{0}^{1} w^{2}\left(\frac{1}{2} w^{2}+c_{V} \theta\right) d x \\
+C^{-1}\left(\int_{0}^{1} \theta_{x}^{2} d x+\int_{0}^{1} w^{2} d x \cdot \int_{0}^{1} w_{x}^{2} d x\right) \\
\leq C\left\{\mathscr{V}(t)+\int_{0}^{1}\left(w^{2} w_{x}^{2}+w^{2}\right) d x\right\} .
\end{aligned}
$$

Here we use several estimates of integration which follow from the aforementioned step, for example

$$
\int_{0}^{1} \theta^{2} d x \cdot \int_{0}^{1} w^{2} d x \leq \max _{x \in[0,1]} \theta(x, t) \cdot \int_{0}^{1} \theta d x \cdot \int_{0}^{1} w^{2} d x \leq C\left(\int_{0}^{1} w^{2} d x+\mathscr{V}(t)\right) .
$$

Similarly we multiply both sides of $(2.6)$ by $w^{3}$, and integrate with respect to $x$ over $[0,1]$. The outcome is

$$
\begin{aligned}
& \frac{1}{4} \frac{d}{d t} \int_{0}^{1} w^{4} d x+\int_{0}^{1}\left(w^{4}+2 \mu \frac{w^{2} w_{x}^{2}}{u}\right) d x \\
& \leq \varepsilon \int_{0}^{1} \theta_{x}^{2} d x+C(\varepsilon)\left(\int_{0}^{1} w^{2} d x+\mathscr{V}(t)\right)
\end{aligned}
$$

for any $\varepsilon>0$. 
Combining (2.34) and (2.35), we find that

$$
\begin{aligned}
\frac{d}{d t} \int_{0}^{1}\left[\left\{\frac{1}{2} w^{2}\right.\right. & \left.\left.+c_{V}\left(\theta-\int_{0}^{1} \theta d x\right)\right\}^{2}+C w^{4}\right] d x \\
+ & k^{*} \int_{0}^{1}\left[\left\{\frac{1}{2} w^{2}+c_{V}\left(\theta-\int_{0}^{1} \theta d x\right)\right\}^{2}+C w^{4}\right] d x \\
+ & C^{-1}\left\{\int_{0}^{1}\left(\theta_{x}^{2}+w^{2} w_{x}^{2}\right) d x+\int_{0}^{1} w^{2} d x \cdot \int_{0}^{1} w_{x}^{2} d x\right\} \\
& \leq C\left(\mathscr{V}(t)+\int_{0}^{1} w^{2} d x\right)
\end{aligned}
$$

holds for some $k^{*}>0$, which gives the assertion for $0<k \leq k^{*}$ by (2.24) and the previous lemma. The assertion for $k>k^{*}$ follows from that for $k=k^{*}$.

3rd Step. $L^{2}(0,1)$ decay of $\left(u_{x}, w_{x}, \theta_{x}\right)$. In a consequence of the foregoing result we have only to show the following proposition.

Proposition 2.3. We have

$$
\int_{0}^{1}\left\{\left(w-\mu \frac{u_{x}}{u}\right)^{2}+z_{x}^{2}+\theta_{x}^{2}\right\} d x=o(1)
$$

where

$$
z(x, t)=\mu w(x, t)+\int_{0}^{x}(\mu u(\xi, t)-R \theta(\xi, t)) d \xi .
$$

Proof. By the help of (2.5), we can rewrite (2.6) as

$$
\frac{\partial}{\partial t}\left(w-\mu \frac{u_{x}}{u}\right)+w-\mu \frac{u_{x}}{u}=\frac{(R \theta-\mu u) u_{x}}{u^{2}}-R \frac{\theta_{x}}{u} .
$$

Multiplying both sides by $w-\mu u_{x} u^{-1}$, we integrate with respect to $x$ over $[0,1]$. By Schwarz's inequality we find that there exists a $T \geq 0$ such that

$$
\begin{aligned}
& \frac{d}{d t} \int_{0}^{1}\left(w-\mu \frac{u_{x}}{u}\right)^{2} d x+\int_{0}^{1}\left(w-\mu \frac{u_{x}}{u}\right)^{2} d x \\
& \leq C\left\{\mathscr{V}(t) \int_{0}^{1}\left(w-\mu \frac{u_{x}}{u}\right)^{2} d x+\int_{0}^{1}\left(w^{2}+\theta_{x}^{2}\right) d x+\mathscr{V}(t)\right\}
\end{aligned}
$$

holds for $t \geq T$. Here we use (2.27) and the fact that for any $\varepsilon>0$,

$$
|R \theta-\mu u| \leq R\left|\theta-\int_{0}^{1} \theta d x\right|+\left|R \int_{0}^{1} \theta d x-\mu u\right| \leq \varepsilon+C(\varepsilon) \mathscr{V}(t)
$$

holds for $t \geq T=T(\varepsilon)$ (see the proof of Lemma 2.3). We integrate the above differential inequality. Because of (2.24) and (2.32), application of Gronwall's lemma gives the $L^{2}$-decay of $w-\mu u_{x} u^{-1}$ and thus that of $u_{x}$.

In order to derive the $L^{2}$-decay of $\left(z_{x}, \theta_{x}\right)$ rigorously, we approximate initial data smoothly such that the solution has derivatives $z_{x t}, \theta_{x t}$ in the classical sense, and 
then pass to the limit. However, since this procedure is rather routine, we shall not give it here.

From (2.5)-(2.7), (2.10), and (2.11), we find that $z$ satisfies the equation

$$
z_{t}+\frac{R}{c_{V}} z=\mu\left(\frac{z_{x}}{u}\right)_{x}-\frac{R}{c_{V}} \int_{0}^{x} \frac{z_{x} w_{x}}{u}(\xi, t) d \xi-\frac{\kappa R}{c_{V}} \frac{\theta_{x}}{u}-\mu\left(1-\frac{R}{c_{V}}\right) w(0, t),
$$

and the boundary condition

$$
z_{x}(0, t)=z_{x}(1, t)=0 .
$$

We multiply both sides of (2.38) by $z_{x x}$, and integrate by parts with respect to $x$ over $[0,1]$ with the help of $(2.39)$. Then it is easy to see that

$$
\frac{1}{2} \frac{d}{d t} \int_{0}^{1} z_{x}^{2} d x+\frac{R}{c_{V}} \int_{0}^{1} z_{x}^{2} d x+C^{-1} \int_{0}^{1} z_{x x}^{2} d x \leq C \int_{0}^{1} \theta_{x}^{2} d x \text { for } t \geq T
$$

holds for some $T(\geq 0)$, if we note that for any $\varepsilon>0$

$$
\left|\int_{0}^{1} z_{x x}(x, t) \int_{0}^{x} \frac{z_{x} w_{x}}{u}(\xi, t) d \xi d x\right|+\left|\int_{0}^{1} \frac{z_{x} z_{x x} u_{x}}{u^{2}} d x\right| \leq \varepsilon \int_{0}^{1} z_{x x}^{2} d x \text { for } t \geq T
$$

holds for some $T=T(\varepsilon) \geq 0$. To see this estimate, we perform an appropriate integration by parts on the left-hand side and use the $L^{2}$-decay of $\left(w, u_{x}\right)$. By virtue of Proposition 2.2, (2.40) gives the $L^{2}$-decay of $z_{x}$ and thus that of $w_{x}$ and

$$
e^{-k t} \int_{0}^{t} e^{k \tau} \int_{0}^{1} z_{x x}^{2} d x d \tau=o(1)
$$

for any $k>0$.

Finally we prove the $L^{2}$-decay of $\theta_{x}$. We multiply both sides of (2.7) by $\theta_{x x}$ and perform a similar procedure to the above argument to have

$$
\frac{d}{d t} \int_{0}^{1} \theta_{x}^{2} d x+C^{-1} \int_{0}^{1}\left(\theta_{x}^{2}+\theta_{x x}^{2}\right) d x \leq C \int_{0}^{1}\left(z_{x}^{2}+z_{x x}^{2}+\theta_{x}^{2}\right) d x \text { for } t \geq T,
$$

which together with (2.32) yields the desired fact.

Now we complete the proof of Theorem 2.1 , and therefore Theorem 1.1 is partially proven.

3. Rate of convergence. In this section $(u, w, \theta)$ is a classical solution to the problem (2.5)-(2.7), (2.9)-(2.11), and $z$ is a function given by (2.36). To prove the remainder of Theorem 1.1, we shall show

THEOREM 3.1. The rate of convergence of $(u, w, \theta)$ to $(\bar{u}, 0, \bar{\theta})$ in $W^{1,2}(0,1)$ is exponential, i.e., there exist positive constants $C, \lambda$ which depend on $R, \mu, c_{V}, \kappa$, and the initial data such that

$$
\int_{0}^{1}\left\{(u-\bar{u})^{2}+w^{2}+(\theta-\bar{\theta})^{2}+u_{x}^{2}+w_{x}^{2}+\theta_{x}^{2}\right\} d x \leq C e^{-\lambda t}
$$

holds.

Outline of Proof. In a consequence of Theorem 2.1, we have

$$
\int_{0}^{1}\left\{(u-\bar{u})^{2}+w^{2}+(\theta-\bar{\theta})^{2}+z^{2}+u_{x}^{2}+w_{x}^{2}+\theta_{x}^{2}+z_{x}^{2}\right\}(x, t) d x \leq \delta
$$


for some $\delta>0$, and, without loss of generality, may assume that $\delta$ is as small as necessary.

For positive constants $C_{i}(i=1, \ldots, 4)$ define $\mathscr{E}(t) \equiv \mathscr{E}\left(t ; C_{1}, C_{2}, C_{3}, C_{4}\right)$ :

$$
\begin{aligned}
\mathscr{E}(t) \equiv \int_{0}^{1}\left[\frac{1}{2} \frac{w^{2}}{u}+\frac{c_{V} \mu}{c_{V}+R}\left(\frac{R \theta}{\mu u}-\log \frac{R \theta}{\mu u}-1\right)+C_{1}\left(w-\mu \frac{u_{x}}{u}\right)^{2}+C_{2} \theta_{x}^{2}+C_{3} z_{x}^{2}\right. \\
\left.+C_{4}\left\{\frac{1}{2} \psi^{2}+c_{V} \bar{\theta}\left(\frac{\theta}{\bar{\theta}}-\log \frac{\theta}{\bar{\theta}}-1\right)\right\}\right] d x,
\end{aligned}
$$

where

$$
\psi(x, t)=w(x, t)+\int_{0}^{x}(u(\xi, t)-\bar{u}) d \xi-\int_{0}^{1} \int_{0}^{\xi}(u(\eta, t)-\bar{u}) d \eta d \xi .
$$

Since both $u$ and $\theta$ are strictly positive and bounded by Theorem $2.1, \mathscr{E}(t)$ is equivalent to $\|(u, w, \theta)\|_{1,2}^{2}$. Consequently we have only to prove that, under suitable choices of $C_{i}$ 's, there exists a positive constant $\lambda$ such that

$$
\frac{d}{d t} \mathscr{E}(t)+\lambda \mathscr{E}(t) \leq 0
$$

holds for $t \geq 0$ if $\delta$ is sufficiently small. We shall give the proof of (3.2) by two lemmas.

Lemma 3.1. If $\delta$ is sufficiently small, then there exist positive constants $C_{i}$ ( $i=$ $1,2,3,5,6)$ such that

$$
\begin{array}{r}
\frac{d}{d t} \int_{0}^{1}\left\{\frac{1}{2} \frac{w^{2}}{u}+\frac{c_{V} \mu}{c_{V}+R}\left(\frac{R \theta}{\mu u}-\log \frac{R \theta}{\mu u}-1\right)+C_{1}\left(w-\mu \frac{u_{x}}{u}\right)^{2}+C_{2} \theta_{x}^{2}+C_{3} z_{x}^{2}\right\} d x \\
+C_{5} \int_{0}^{1}\left\{w^{2}+\left(\frac{R \theta}{\mu u}-\log \frac{R \theta}{\mu u}-1\right)+\left(w-\mu \frac{u_{x}}{u}\right)^{2}+w_{x}^{2}+z_{x}^{2}+\theta_{x x}^{2}+z_{x x}^{2}\right\} d x \\
\leq C_{6} \int_{0}^{1} \theta_{x}^{2} d x
\end{array}
$$

holds.

Proof. We multiply both sides of $(2.6)$ by $u^{-1} w$, and integrate with respect to $x$ over $[0,1]$ by use of $(2.10)$. Then we get

$$
\begin{gathered}
\frac{d}{d t} \int_{0}^{1}\left(\frac{1}{2} \frac{w^{2}}{u}+\mu \log u\right) d x+\int_{0}^{1}\left(\frac{w^{2}}{u}+\mu \frac{w_{x}^{2}}{u^{2}}\right) d x \\
=\int_{0}^{1}\left\{R \frac{\theta w_{2}}{u^{2}}+\frac{w u_{x} z_{x}}{u^{3}}-\frac{1}{2} \frac{w^{2} w_{x}}{u^{2}}\right\} d x .
\end{gathered}
$$

Here we use also (2.5). On the other hand, (2.5) and (2.7) yield $\int_{0}^{1} \frac{\theta w_{x}}{u^{2}} d x=-\frac{d}{d t} \int_{0}^{1} \frac{\theta}{u} d x+\frac{1}{c_{V}} \int_{0}^{1}\left\{\frac{z_{x}}{u}-R \frac{\theta w_{x}}{u^{2}}+\mu \frac{w_{x}^{2}}{u^{2}}+\mu \frac{w_{x}}{u}+\kappa\left(\frac{\theta_{x}}{u}\right)_{x} \frac{1}{u}\right\} d x$. By the help of (2.2), (2.4), (2.36), and (2.12), we write $u^{-1} z_{x}$ as $\left(\int_{0}^{x} v d \xi\right)_{t}$ and $u^{-1} w_{x}$ as $(\log u)_{t}$. By use of (2.7) we perform integration by parts of the last term of the 
above equality. From this result, (3.4), and (2.23), we get

$$
\begin{aligned}
& \frac{d}{d t} \int_{0}^{1}\left\{\frac{1}{2} \frac{w^{2}}{u}+\frac{c_{V} \mu}{c_{V}+R}\left(\frac{R \theta}{\mu u}-\log \frac{R \theta}{\mu u}-1\right)\right\} d x \\
& \quad+\int_{0}^{1}\left(\frac{w^{2}}{u}+\frac{c_{V} \mu}{c_{V}+R} \frac{w_{x}^{2}}{u^{2}}+\frac{1}{c_{V}+R} \frac{z_{x}^{2}}{u \theta}+\frac{\kappa \mu}{c_{V}+R} \frac{\theta_{x}^{2}}{u \theta^{2}}\right) d x \\
& =\int_{0}^{1}\left(\frac{w u_{x} z_{x}}{u^{3}}-\frac{1}{2} \frac{w^{2} w_{x}}{u^{2}}+\frac{\kappa R}{c_{V}+R} \frac{\theta_{x} u_{x}}{u^{3}}\right) d x \\
& \leq C \delta^{1 / 2} \int_{0}^{1}\left\{\left(w-\mu \frac{u_{x}}{u}\right)^{2}+w^{2}+w_{x}^{2}+z_{x}^{2}\right\} d x \\
& \quad+\varepsilon \int_{0}^{1}\left\{\left(w-\mu \frac{u_{x}}{u}\right)^{2}+w^{2}\right\} d x+C(\varepsilon) \int_{0}^{1} \theta_{x}^{2} d x .
\end{aligned}
$$

On the other hand, since $R \theta / \mu u$ is strictly positive and bounded,

$$
0 \leq \frac{R \theta}{\mu u}-\log \frac{R \theta}{\mu u}-1 \leq C(R \theta-\mu u)^{2} \leq C\left(z_{x}^{2}+w_{x}^{2}\right)
$$

holds.

Moreover, (2.37), (2.40), and (2.41) hold for $t \geq 0$ if $\delta$ is sufficiently small. Combining these estimates and (3.5)-(3.6), and taking $\delta$ and $\varepsilon$ sufficiently small, we get (3.3) for some $C_{i}$ 's.

If an inequality $C_{6}<C_{5}$ holds, then we can easily show the exponential $L^{2}$-decay of $\left(w, R \theta-\mu u, z, u_{x}, w_{x}, \theta_{x}, z_{x}\right)$ by use of (3.3). The inequality is true when $R / c_{V}$ is sufficiently small, see $[5,1]$. In general, however, one cannot expect it. Therefore we need a more delicate analysis.

LEMMA 3.2. We have

$$
\begin{gathered}
\frac{d}{d t} \int_{0}^{1}\left\{\frac{1}{2} \psi^{2}+c_{V} \bar{\theta}\left(\frac{\theta}{\bar{\theta}}-\log \frac{\theta}{\bar{\theta}}-1\right)\right\} d x+\bar{\theta} \int_{0}^{1}\left(\frac{z_{x}^{2}}{\mu u \theta}+\kappa \frac{\theta_{x}^{2}}{u \theta^{2}}\right) d x=0, \\
\int_{0}^{1}\left\{\psi^{2}+\psi_{x}^{2}+\left(\frac{\theta}{\bar{\theta}}-\log \frac{\theta}{\bar{\theta}}-1\right)\right\} d x \leq C \int_{0}^{1}\left\{w^{2}+\left(w-\mu \frac{u_{x}}{u}\right)^{2}+w_{x}^{2}+\theta_{x}^{2}+z_{x}^{2}\right\} d x .
\end{gathered}
$$

Proof. Using $z(x, t)$ and $\psi(x, t)$, we can rewrite (2.6) and (2.7) as

$$
\psi_{t}=\left(\frac{z_{x}}{u}\right)_{x}
$$

and

$$
c_{V} \theta_{t}=\frac{z_{x} \psi_{x}}{u}+\frac{\bar{u} z_{x}}{u}+\kappa\left(\frac{\theta_{x}}{u}\right)_{x}
$$

or

$$
c_{V} \theta_{t}=\frac{z_{x}^{2}}{u}+\frac{R \theta z_{x}}{\mu u}+\kappa\left(\frac{\theta_{x}}{u}\right)_{x}
$$

respectively. We multiply the first equation by $\psi$ and the third by $-\bar{\theta} \theta^{-1}$. We sum up these three relations and integrate with respect to $x$ over $[0,1]$ by use of $(2.11)$ and (2.39). If we note (1.8), then we get (3.7). 
Recalling (2.29) and (3.1), we obtain

$$
\int_{0}^{1} \psi d x \equiv 0
$$

Therefore, if we note that

$$
\mu \psi_{x}=z_{x}+R(\theta-\bar{\theta})
$$

holds, we get

$$
\int_{0}^{1} \psi^{2} d x \leq \int_{0}^{1} \psi_{x}^{2} d x \leq C \int_{0}^{1}\left\{z_{x}^{2}+(\theta-\bar{\theta})^{2}\right\} d x .
$$

Moreover, since $\theta$ is bounded and strictly positive,

$$
0 \leq \frac{\theta}{\bar{\theta}}-\log \frac{\theta}{\bar{\theta}}-1 \leq C(\theta-\bar{\theta})^{2}
$$

holds. Consequently, to prove (3.8) we must estimate $\theta-\bar{\theta}$.

$$
(\theta-\bar{\theta})^{2} \leq C\left[\left(\frac{R^{2} \theta}{2 \mu}+6 c_{V} \mu\right)^{2}-\left\{36 c_{V}^{2} \mu^{2}+3 R^{2}\left(2 E_{0}-\left(\int_{0}^{1} v_{0} d x\right)^{2}\right)\right]^{2}\right.
$$

holds by (1.9). From (2.14), (2.4), and (2.29), we have

$$
\begin{aligned}
& 2 E_{0}-\left(\int_{0}^{1} v_{0} d x\right)^{2}=2 c_{V} \int_{0}^{1} \theta d x \\
+ & \int_{0}^{1}\left\{w^{2}+\left(\int_{0}^{x} u d \xi-\int_{0}^{1} \int_{0}^{\xi} u d \eta d \xi\right)^{2}+2 w\left(\int_{0}^{x} u d \xi-\int_{0}^{1} \int_{0}^{\xi} u d \eta d \xi\right)\right\} d x .
\end{aligned}
$$

We insert this into the right-hand side of (3.9). Hence one gets

$$
\begin{gathered}
(\theta-\bar{\theta}) \leq C\left[\frac{R^{4} \theta^{2}}{4 \mu^{2}}+6 R^{2} c_{V}\left(\theta-\int_{0}^{1} \theta d x\right)\right. \\
-3 R^{2} \int_{0}^{1}\left\{w^{2}+\left(\int_{0}^{x} u d \xi-\int_{0}^{1} \int_{0}^{\xi} u d \eta d \xi\right)^{2}\right. \\
\left.\left.+2 w\left(\int_{0}^{x} u d \xi-\int_{0}^{1} \int_{0}^{\xi} u d \eta d \xi\right)\right\} d x\right]^{2} \\
\leq C\left\{\left|\frac{R^{4} \theta^{2}}{4 \mu^{2}}-3 R^{2}\left(\int_{0}^{1} u d x\right)^{2} \int_{0}^{1}\left(x-\frac{1}{2}\right)^{2} d x\right|^{2} \int_{0}^{1}\left(\theta_{x}^{2}+u_{x}^{2}+w^{2}\right) d x\right\} \\
\leq C\left\{(R \theta-\mu u)^{2}+\int_{0}^{1}\left(w^{2}+u_{x}^{2}+\theta_{x}^{2}\right) d x\right\} \\
\leq C\left[z_{x}^{2}+w_{x}^{2}+\int_{0}^{1}\left\{w^{2}+\left(w-\mu \frac{u_{x}}{u}\right)^{2}+\theta_{x}^{2}\right\} d x\right] .
\end{gathered}
$$


Thus the lemma is valid.

It easily follows from Lemmas 3.1 and 3.2 that (3.2) is valid for some $C_{i}$ 's. Therefore we complete the proof of Theorem 3.1. By use of the original time variable and unknown functions, Theorem 1.1 is completely proven from Theorems 2.1 and 3.1.

Acknowledgment. The author would like to thank Professor Shuichi Kawashima, of Kyushu University, for valuable discussions.

\section{REFERENCES}

[1] S. Kawashima, Large-time behavior of solutions to the free boundary problem for the equations of a viscous heat-conductive gas, preprint

[2] A. V. Kazhykhov, Sur le solubilité globale des problèmes monodimensionnels aux valeurs initialeslimitées pour les équations d'un gaz visqueux et calorifere, C. R. Acad. Sci. Paris Sér. A. 284, 317-320 (1977)

[3] T. Nagasawa, On the one-dimensional motion of the polytropic ideal gas non-fixed on the boundary, J. Differential Equations 65, 49-67 (1986)

[4] T. Nagasawa, On the outer pressure problem of the one-dimensional polytropic ideal gas, Japan J. Appl. Math. 5, 53-85 (1988)

[5] M. Okada, Free boundary value problems for the equations of one-dimensional motion of compressible viscous fluids, Japan J. Appl. Math. 4, 219-235 (1987) 